\title{
Increasing the Efficacy of Stem Cell Therapy via Triple-Function Inorganic Nanoparticles
}

Fang Chen ${ }^{1,2}$, Eric Ruike Zhao ${ }^{1}$, Ghanim Hableel ${ }^{1}$, Tao Hu ${ }^{3}$, Taeho Kim ${ }^{1,4}$, Jingting $\mathrm{Li}^{5}$, Natalia Isabel Gonzalez-Pech ${ }^{6}$, David J. Cheng ${ }^{1}$, Jeanne E. Lemaster ${ }^{1}$, Yijun Xie $^{2,6}$, Vicki H. Grassian ${ }^{1,6,7}$, George L. Sen ${ }^{5}$, Jesse V. Jokerst ${ }^{1,2,8, *}$

${ }^{1}$ Department of NanoEngineering, University of California San Diego, 9500 Gilman Drive, La Jolla, CA 92093, USA

${ }^{2}$ Materials Science and Engineering Program, University of California San Diego, 9500 Gilman Drive, La Jolla, CA 92093, USA

${ }^{3}$ School of Materials Science and Engineering, Central South University, Changsha 410083, China

${ }^{4}$ Department of Biomedical Engineering, Institute of Quantitative Health Science and Engineering, Michigan State University, 775 Wood lot Drive, East Lansing, MI 48824, USA

${ }^{5}$ Department of Dermatology and Cellular and Molecular Medicine, University of California San Diego, 9500 Gilman Drive, La Jolla, CA 92093, USA

${ }^{6}$ Department of Chemistry and Biochemistry, University of California, San Diego, 9500 Gilman Drive, La Jolla, CA 92093, USA 
${ }^{7}$ Scripps Institution of Oceanography, San Diego, 9500 Gilman Drive, La Jolla, CA 92093, USA

${ }^{8}$ Department of Radiology, University of California, San Diego, 9500 Gilman Drive, La Jolla, CA 92093, USA

* Corresponding author: jjokerst@eng.ucsd.edu 


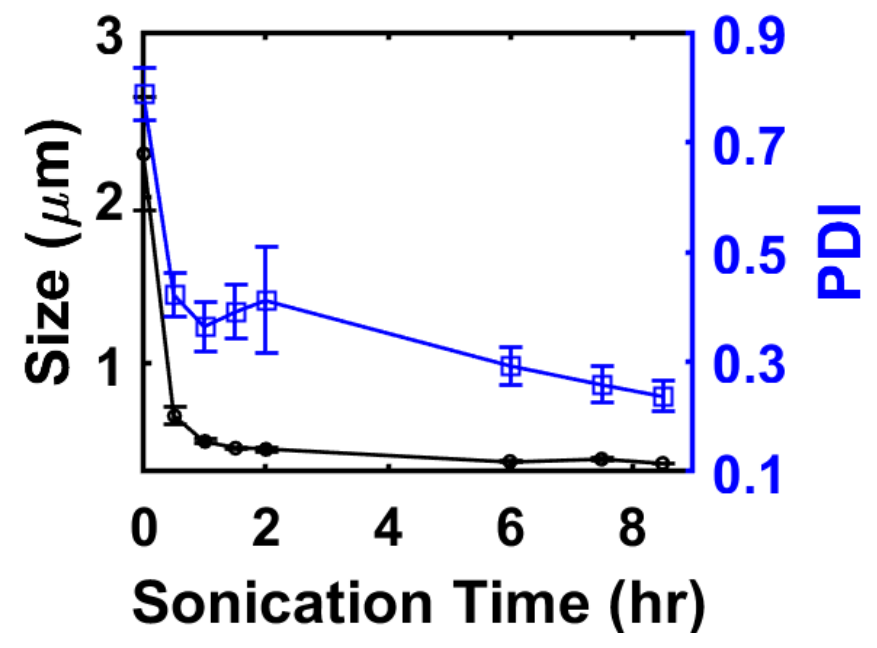

Figure S1. Size and PDI of MCF. Both average DLS size and polydispersity index of MCF decrease with increasing sonication time. The error bars represent the re standard deviation of three measurements.

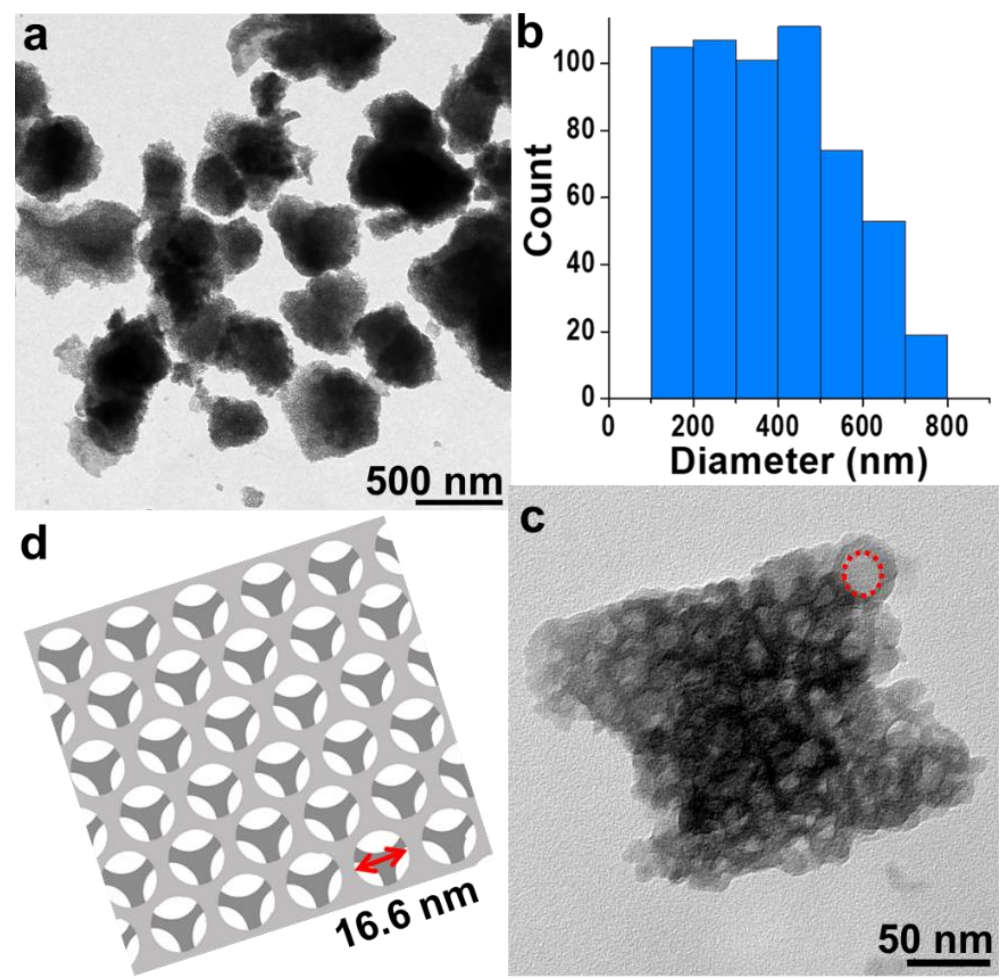

Figure S2. Characterization of optimized MCF. (a) TEM image and (b) size distribution ( $\mathrm{n}=570)$ of MCF sonicated for 8.5 hours. The diameter is the average of Feret and MinFeret measured by ImageJ. Feret diameter is the longest distance between any two points along the selection boundary also known as the maximum caliper. MinFeret diameter is the shortest longest distance between any two points along the selection boundary. (c) Higher magnification TEM image shows the 3-dimensional porous structure of MCF. (d) Schematic illustration of MCF porous structure. $^{1}$ 

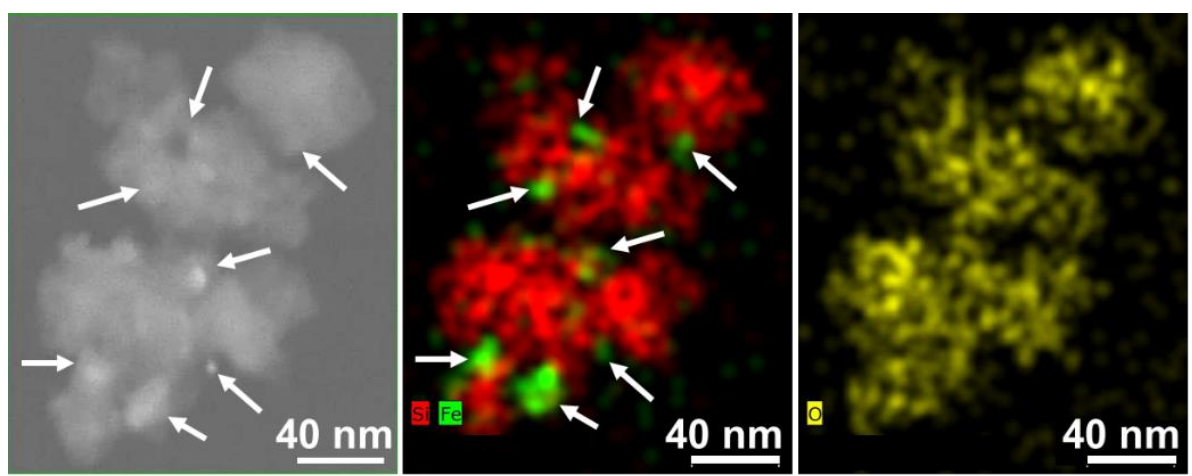

Figure S3. STEM image and EDX mapping of product made by loading pre-formed SPIO into MCF. Grey is the Z-contrast image. Red is silicon, green is iron, and yellow is oxygen. The white arrows indicate the SPIO blockades.

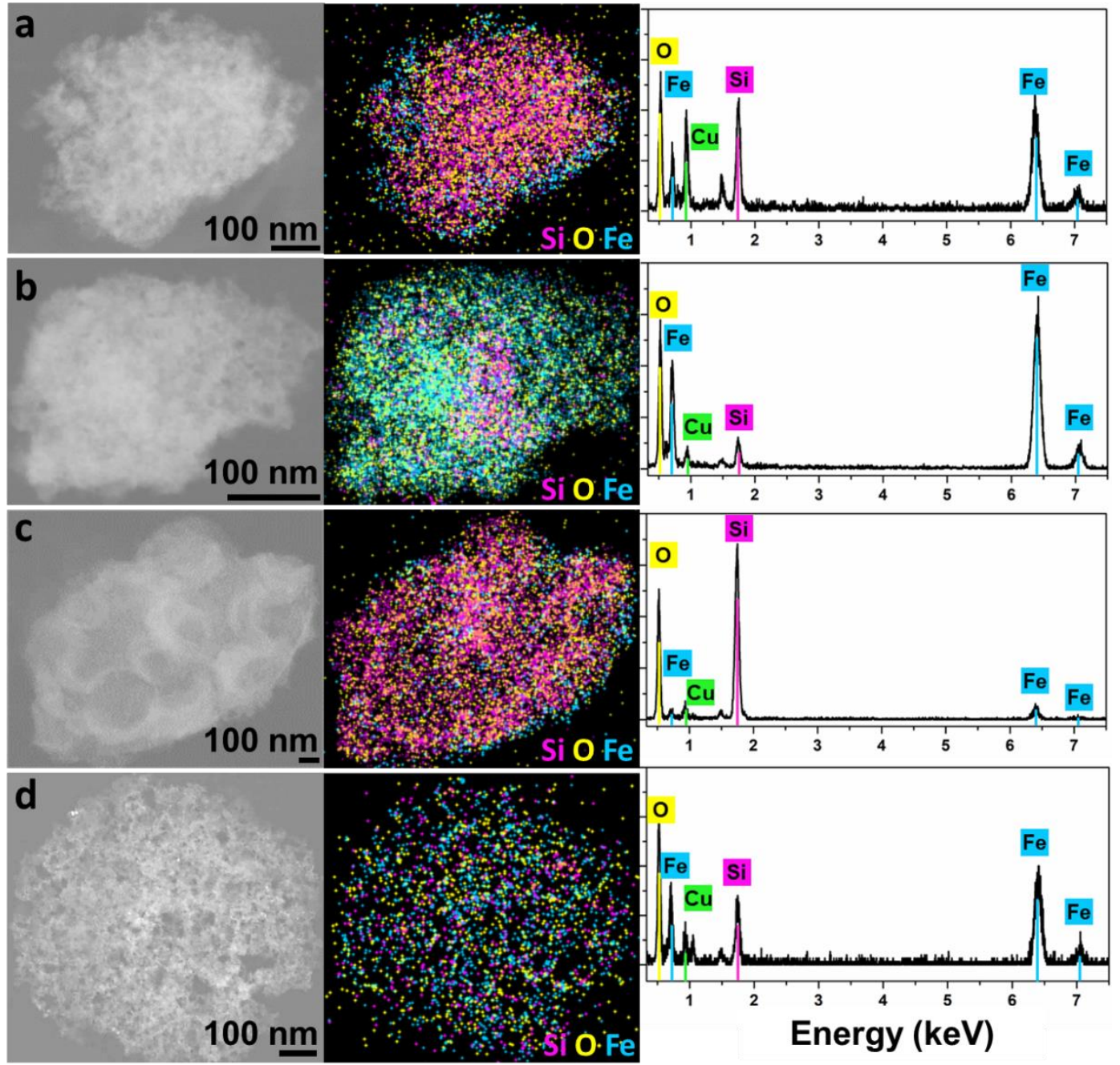

Figure S4. EDX mapping and spectra of SIO under different reaction conditions: (A) $0.7 \mathrm{M}$ ammonia, 60 minutes, RT; (B) $0.5 \mathrm{M}$ sodium hydroxide, 10 minutes, $100^{\circ} \mathrm{C}$; (C) $0.5 \mathrm{M}$ sodium hydroxide, 30 minutes, RT; (D) $0.5 \mathrm{M}$ sodium hydroxide, 60 minutes, RT. The last condition was chosen as an optimized reaction condition. Pink, yellow, cyanine, and green indicate silicon, oxygen, iron, and copper, respectively. 


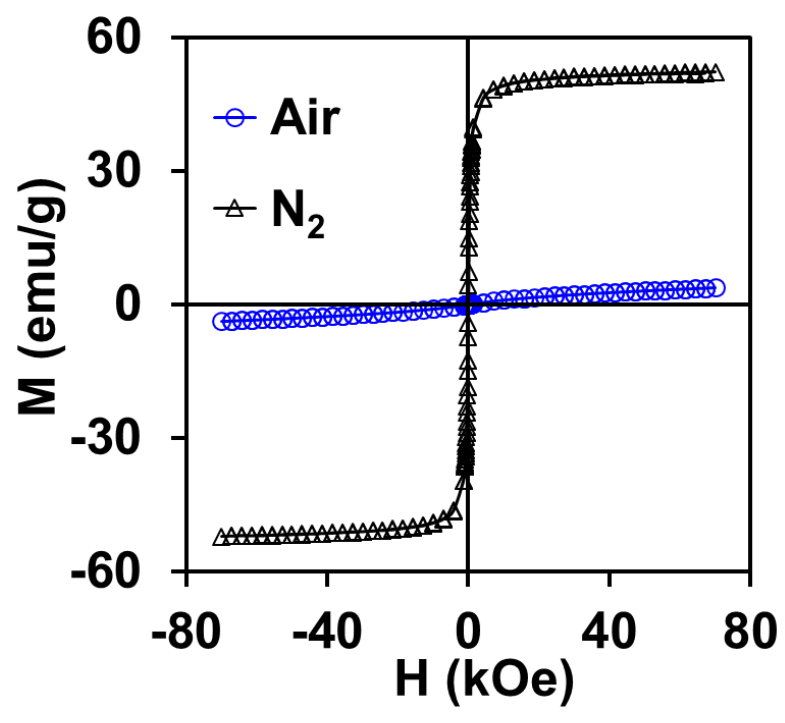

Figure S5. Hysteresis loop of SIO. Hysteresis loop indicates that SIO are superparamagnetic. The nitrogen protection during in situ growth of SPIO significantly increased the saturation magnetization of SIO. The measurement temperature was $300 \mathrm{~K}$.
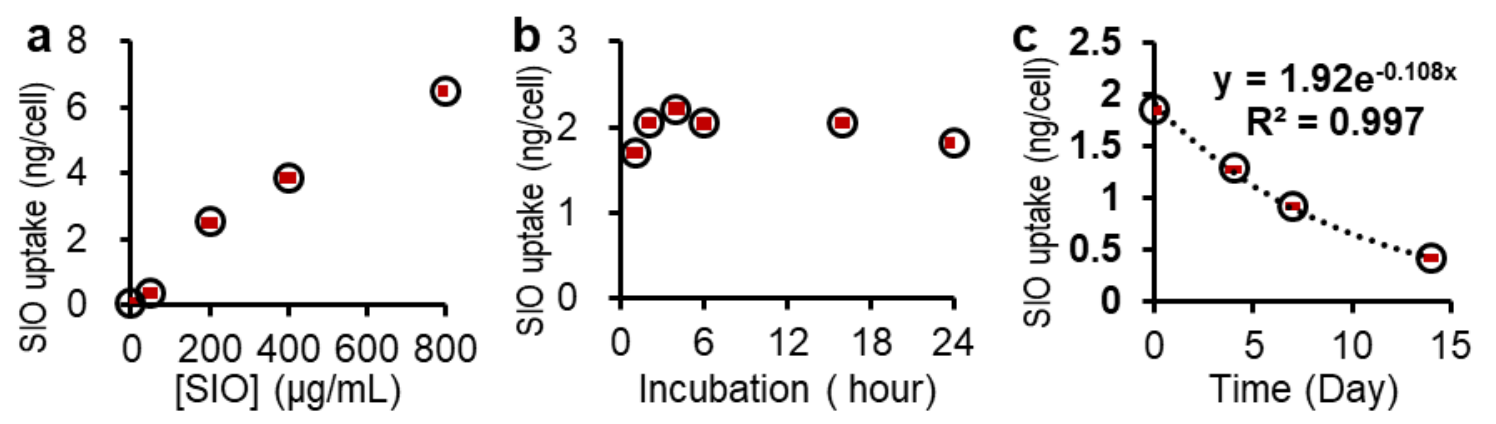

Figure S6. Effect of labeling condition and half-life of nanoparticles inside hMSCs. (a) Loaded SIO increased as the incubation concentration of SIO increased. Error bars are standard deviations of six measurements. (b) The incubation time changed the amount of loaded SIO slightly, with a peak at 4 hours. Error bars are standard deviations of six measurements. (c) Loaded SIO decreased as time went, which is likely due to the division of cells. According to the exponential fitting curve, the half-life of SIO in hMSCs was around 6.7 days. Error bars are standard deviations of three replicates. 


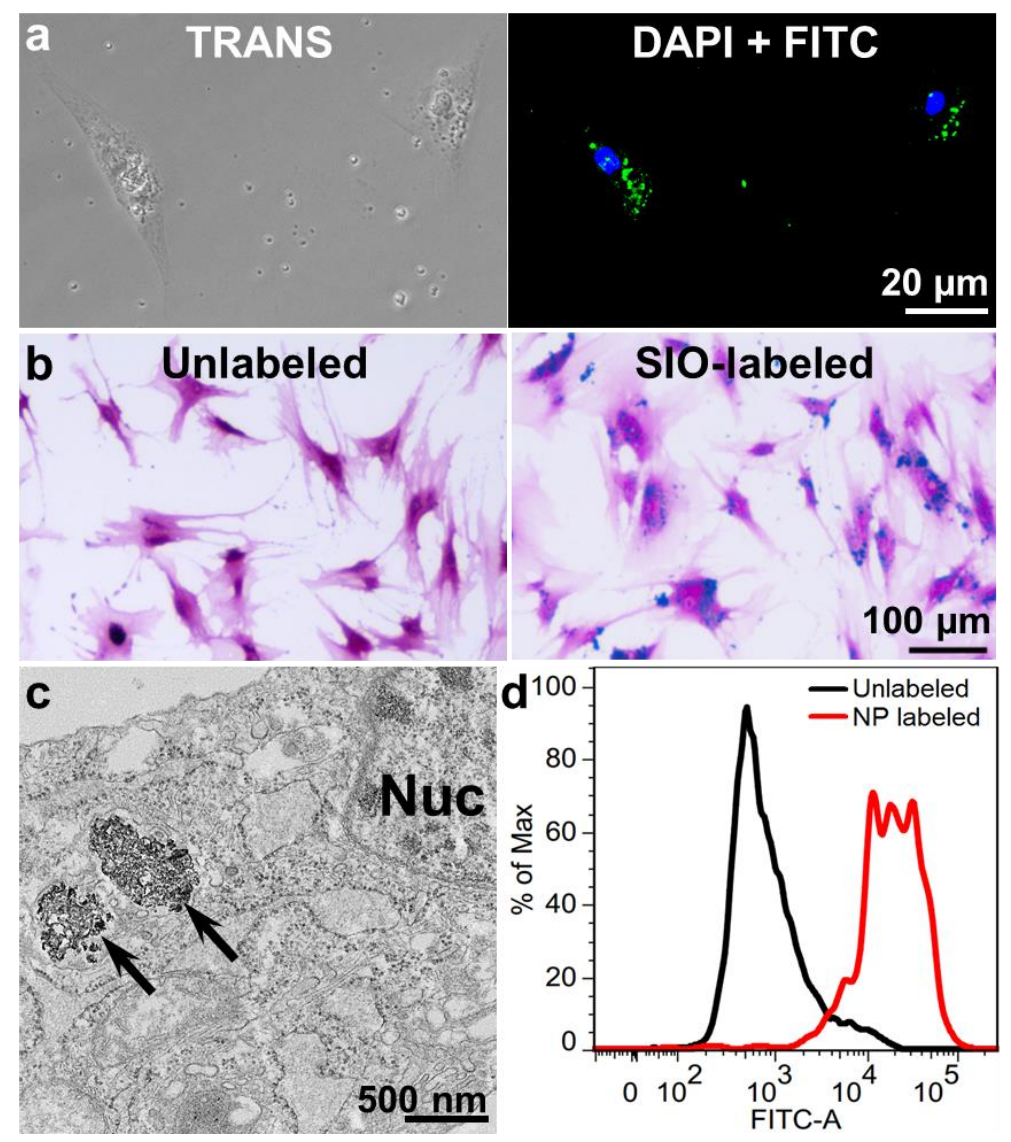

Figure S7. SIO enter and label hMSCs. (a) Photomicrographs of SIO-labeled hMSCs show the presence of FITC-conjugated SIO (green) together with hMSC. Blue indicates the hMSC nuclei. (b) Microscope images show unlabeled and SIO-labeled hMSCs stained with potassium ferrocyanide and pararosaniline solutions (iron staining kit from Sigma Aldrich). Blue, pink, and red indicate the presence of iron, cytoplasm, and nuclei respectively. (c) Sectioning TEM image of SIO-labeled hMSC indicates the presence of SIO in cytoplasm. (d) Flow cytometry shows more than $92.5 \%$ of hMSCs were labeled with FITC-conjugated SIO (red solid line). 

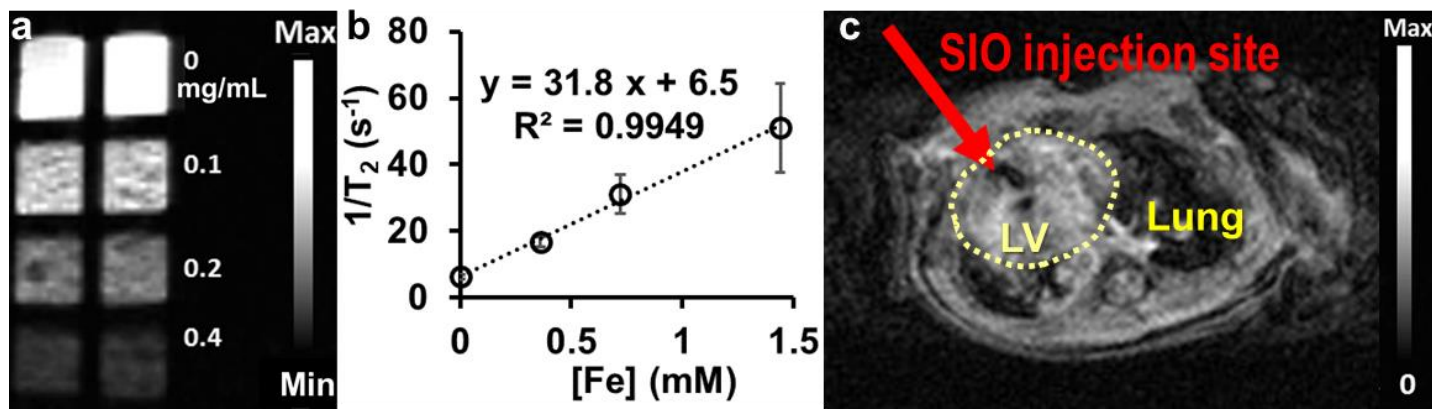

Figure S8. MRI contrast of SIO. (a) MRI of SIO in agarose phantom with different concentrations. Two columns are two replicates. The scan was performed with repetition time $5,000 \mathrm{~ms}$ and echo time $12.6 \mathrm{~ms}$ at $4.7 \mathrm{~T}$. (b) Relaxivity of SIO per Fe was found to be $31.8 \mathrm{mM}^{-1} \mathrm{~s}^{-1}$. Error bars are standard deviations of two SIO batches. (c) In vivo MRI image showed a high MRI contrast of SIO against myocardium.
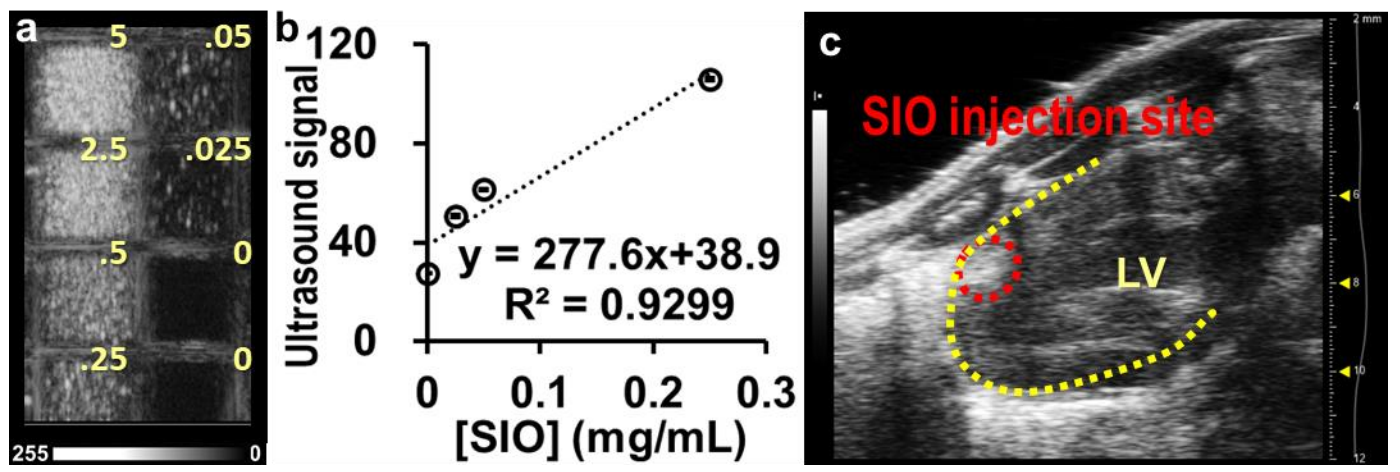

Figure S9. Ultrasound contrast of SIO. (a) Ultrasound image of SIO in agarose phantom with different concentrations from 0 to $5 \mathrm{mg} / \mathrm{mL}$. The scan was performed a linear transducer (MS550, VisualSonics) with $40 \mathrm{MHz}$. (b) Relationship between ultrasound signal and SIO concentration. Error bars are standard deviations of five fields-of-view. (c) In vivo ultrasound images showed the contrast of SIO against myocardium. 


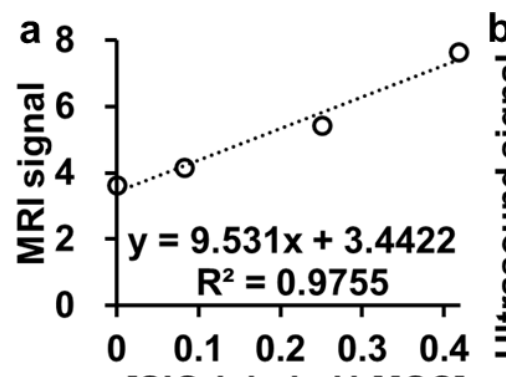

[SIO-labeled hMSC]

(million/mL)

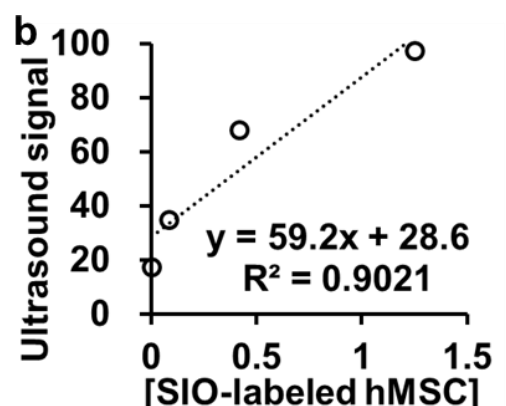

(million/mL)

Figure S10. MRI and US signal of SIO-labeled hMSCs. (a) MRI signals of SIO-labeled hMSCs in agarose phantom was linearly dependent on cell concentrations. (b) Relationship between ultrasound signals and concentration of SIO-labeled hMSCs in agarose phantom.
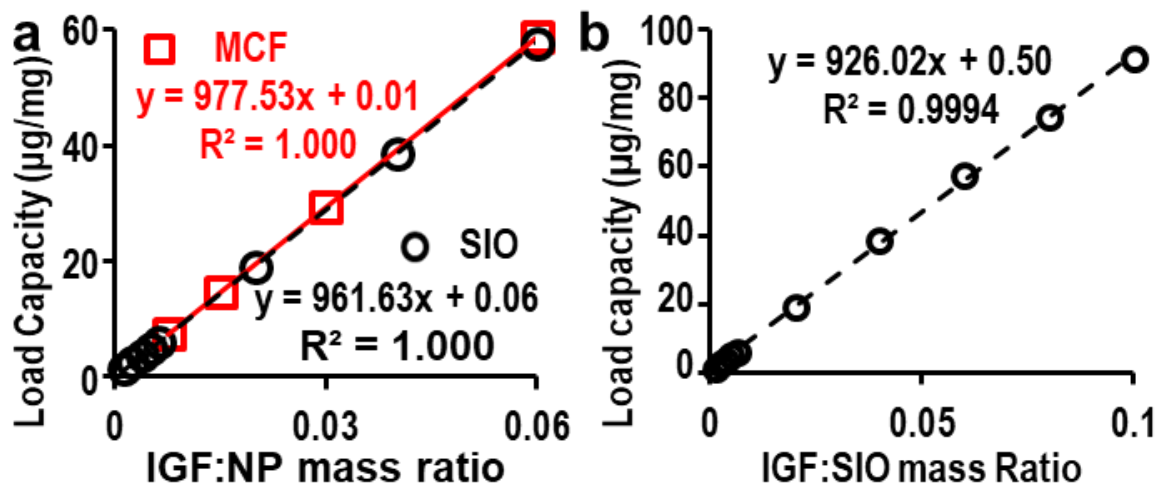

Figure S11. Loading capacity of the MCF and SIO. (a) The loading capacities of both the MCF and SIO showed a linear dependence on the mass ratio of IGF :nanoparticles. The loading capacity between these two nanoparticles were similar. (b) Loading capacities of SIO showed a linear dependence on the mass ratio of IGF :nanoparticles within $0-0.1$. 


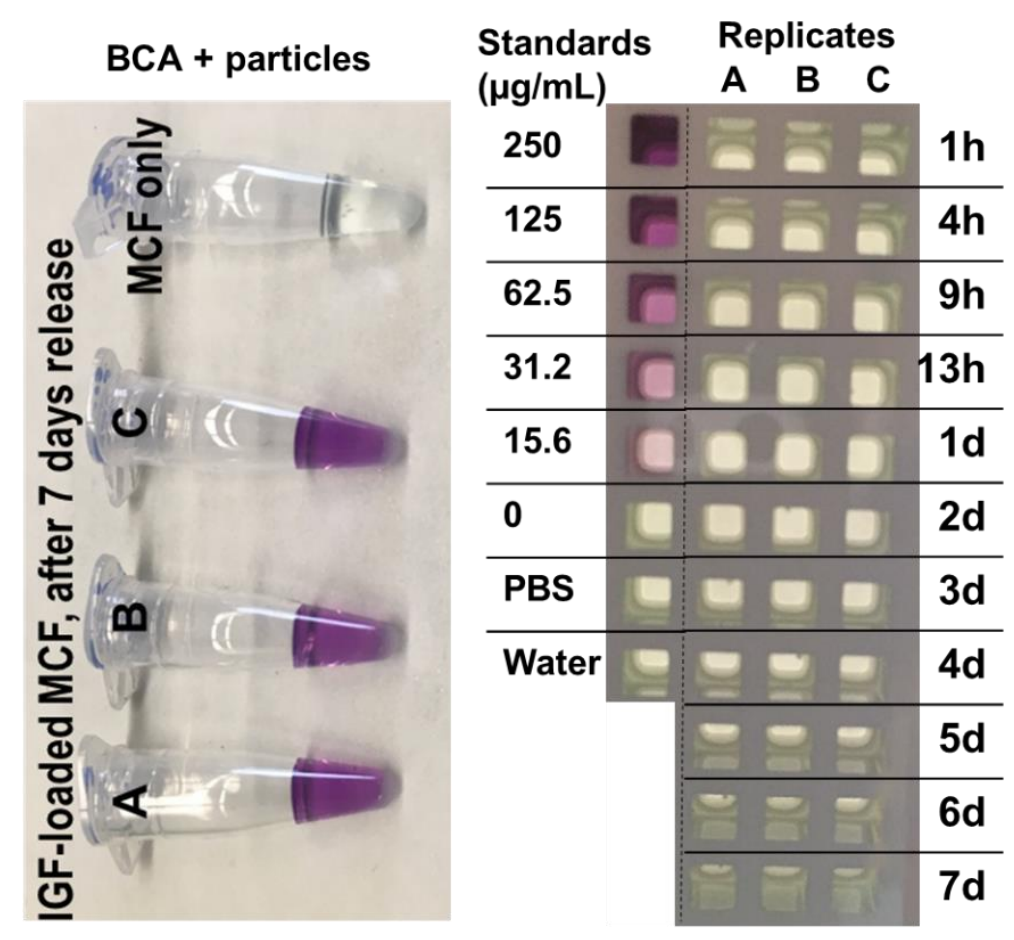

Figure S12. No IGF release from IGF-loaded MCF. The BCA assay produces a purple color in the presence of protein. No IGF release was seen from MCF. After one week of incubation, the IGF-loaded MCF turned when stained with the BCA agent (Left), however, no IGF was detected in the release media. 


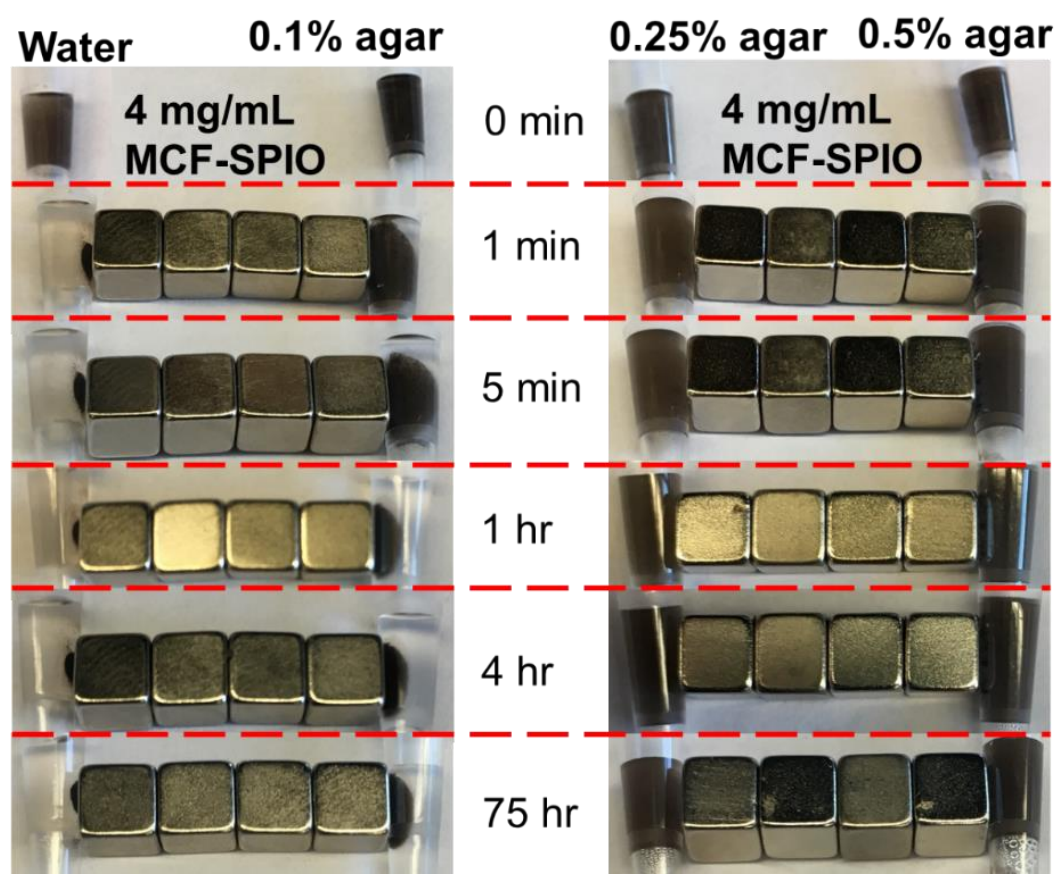

Figure S13. Demonstration of SIO retention in mimicking tissues. The maximum pull force of the cubic magnets is about 10 newtons. Almost all the nanoparticles were pulled out from water within one minute and from $0.1 \%$ agarose within 1 hour. However, the retention of $0.25 \%$ and $0.5 \%$ agarose is strong, and the nanoparticles cannot be pulled out by these magnets. The $0.5 \%$ agarose gel has a similar mechanic al properties as soft tissues. ${ }^{2}$
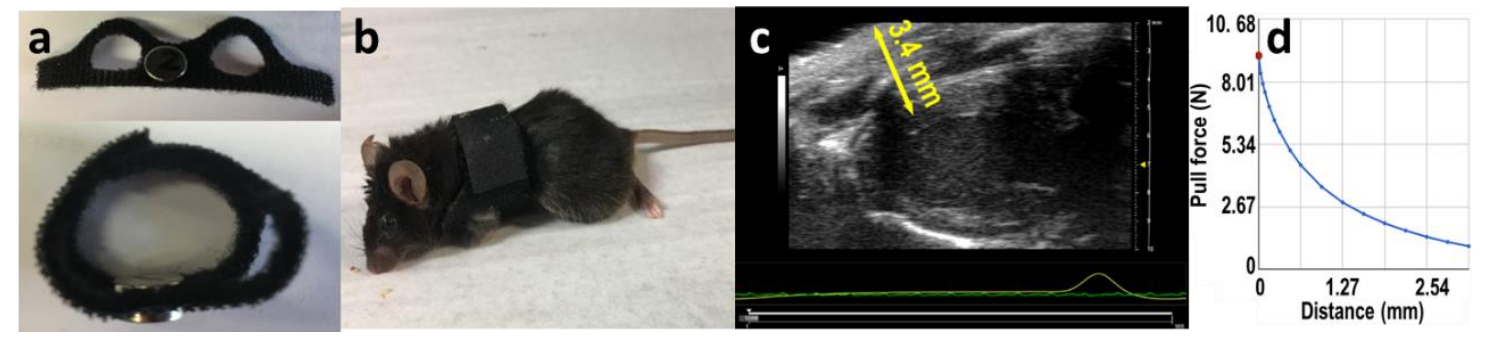

Figure S14. In vivo study of SIO retention at mouse heart apex by external magnet harness. (a) Design of the magnetic harness. Total weight of each harness is controlled within $10 \%$ of the mouse body weight. (b) Mouse wearing a magnetic harness. (c) Ultrasound image indicates the distance between the magnet and apex is approximately $3.4 \mathrm{~mm}$. (d) The pull force of the magnet as a function of distance (adopted from K\&J Magnetics). 


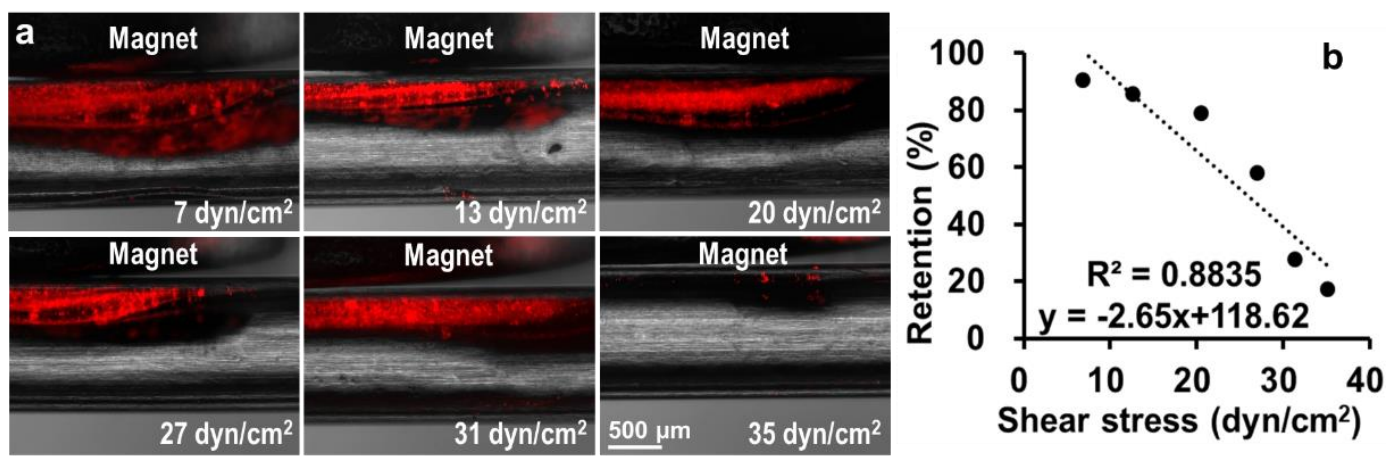

Figure S15. Retention of SIO-labeled hMSCs versus shear stress. (a) Overlay of fluorescence and microscope images show the retention of SIO-labeled hMSCs with the presence of magnet in laminar flow with a range of shear stress $7-35 \mathrm{dyn} / \mathrm{cm}^{2}$. The mean wall shear stress in left ventricle of human is $12.8 \mathrm{dyn} / \mathrm{cm}^{2}{ }^{3}$ Cells were stained with fluorescent quantum dots for visualization. (b) Retention of SIO-labeled hMSCs was dependent on the shear stress.

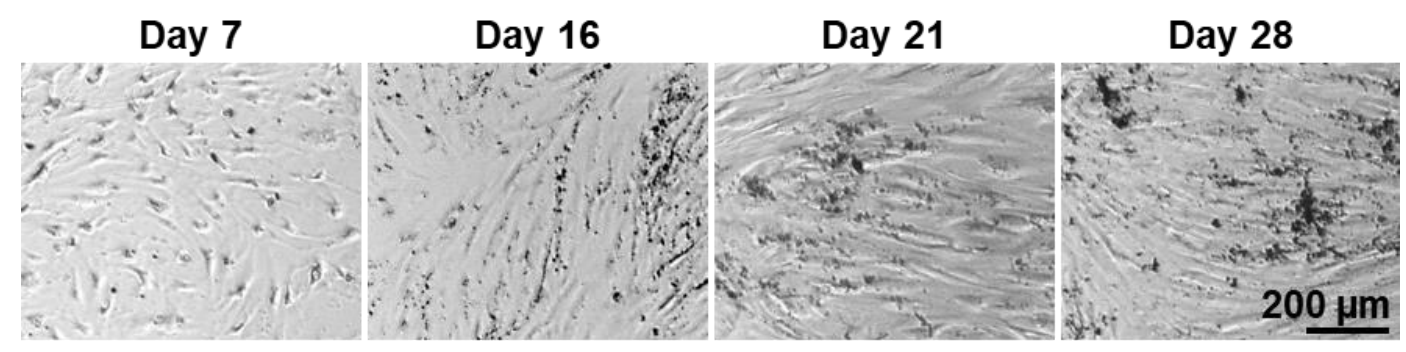

Figure S16. Growth of SIO-labeled hMSCs on the side wall of a flask. The SIO-labeled cells were attracted by an external magnet and started to adhere on the side wall of flask on the first day. The cells continued growing after removal of the magnet. 


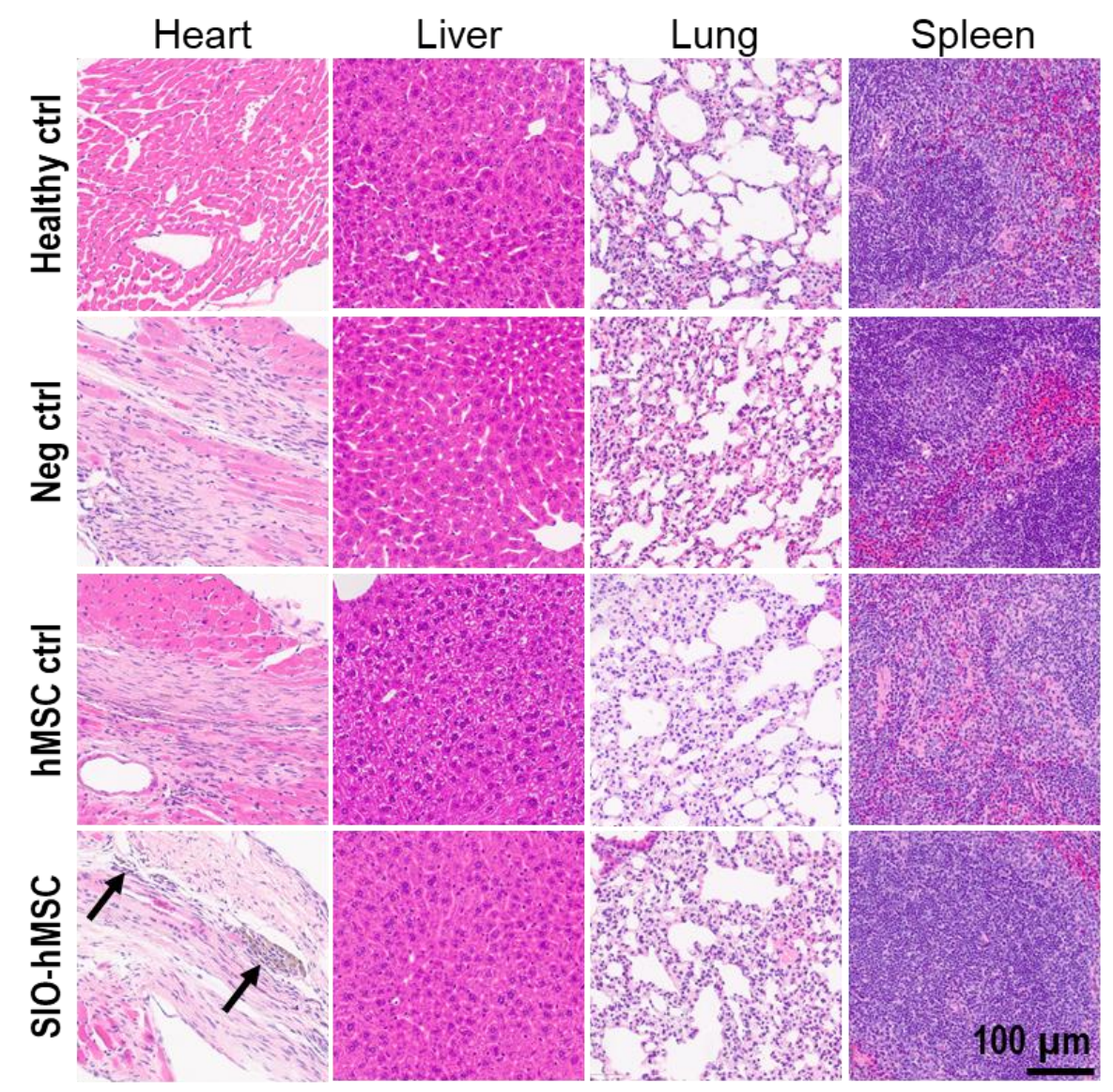

Figure S17. H\&E staining of organs from four groups. Arrows point at brown segments which indicate the presence of SIO. No observable differences were found in liver, lung, and spleen among the four groups. 


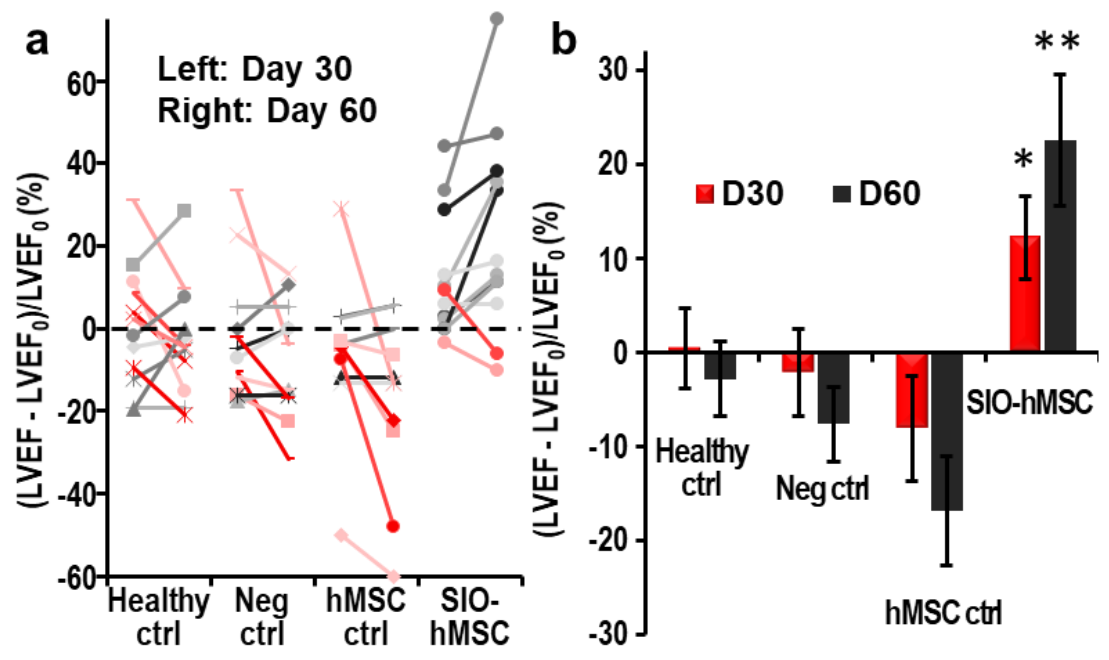

Figure S18. Change of LVEF for individual animals. (a) Change in LVEF from baseline for individual subjects ( $\mathrm{n}=12$ for all four groups). Grey and red indicates the increase and decrease of $\triangle \mathrm{LVEF}$ on day 60 compared to day 30. (b) Mean change in LVEF of each group show that the IGF-loaded SIO-labeled hMSC had significantly improved heart function compared to the other groups on both day 30 and 60 . The asterisks showed the significance in change compared to negative control group. Error bars are standard errors $(n=12){ }^{*} \mathrm{p}<0.05, * * \mathrm{p}<0.005$ (two-tail and type two t-test was used).
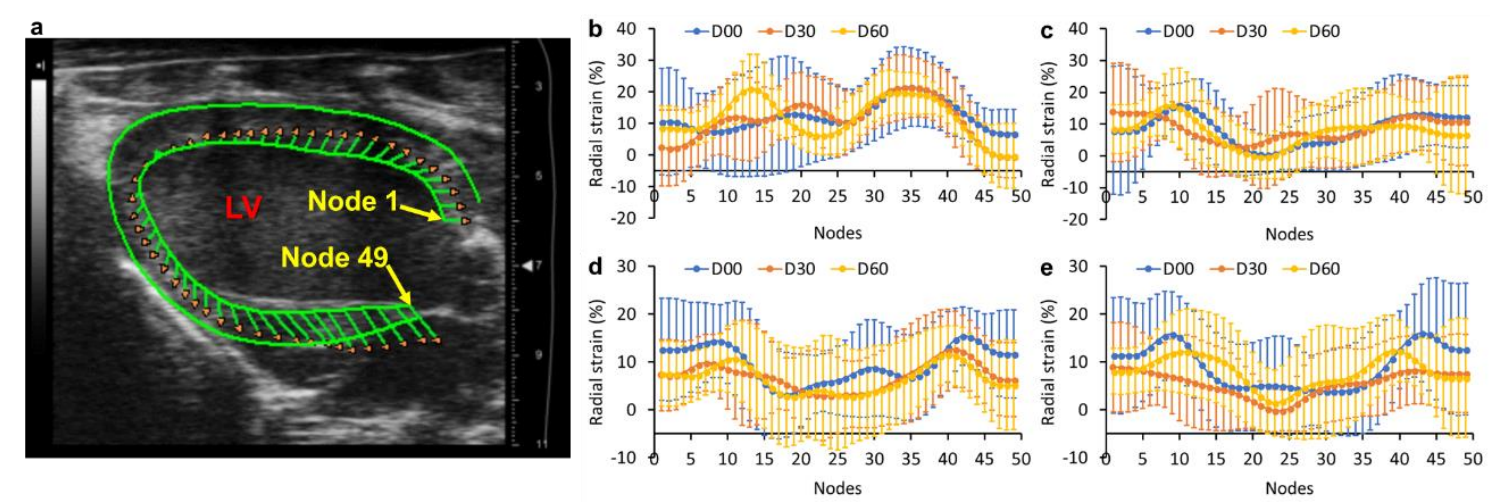

Figure S19. Radial strain analysis. (a) Schemes of the 49 nodes on the LV myocardium. Average radial strain of each node for (b) healthy control group, (c) negative control group, (d) hMSC control group, and (e) IGF-loaded SIO-labeled hMSC treatment group. Error bars are standard deviations $(n=12)$. 


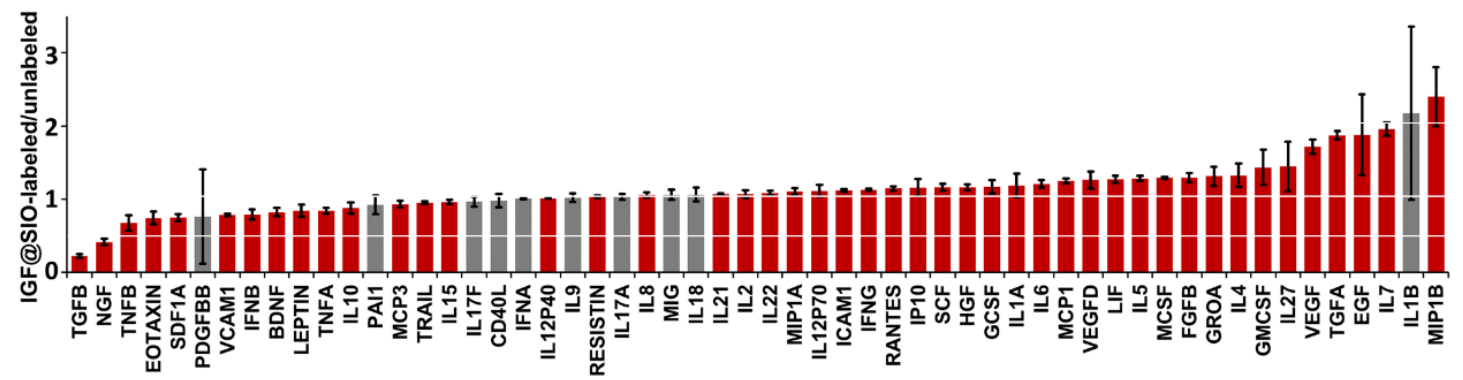

Figure S20. Effect of IGF-loaded SIO on secretome of hMSCs. Fold change of cytokines secreted by IGF-loaded SIO-labeled and unlabeled hMSCs. Cytokines secreted by the IGF-loaded SIO-labeled cells were within 1-fold (50\% - 200\%) of unlabeled cells, except TGFB, NGF, MIP1B, and IL1B. Grey bars indicate a statistically non-significant $(\mathrm{P}>0.05)$ change in expression; red bars indicate significance $(\mathrm{P}<0.05)$, two-tailed homoscedastic test. Error bars are standard deviation of four replicates. 


\section{Reference}

1. Schmidt-Winkel, P.; Lukens, W. W.; Zhao, D.; Yang, P.; Chmelka, B. F.; Stucky, G. D. Mesocellular Siliceous Foams with Uniformly Sized Cells and Windows. J. Am. Chem. Soc. 1999, 121, 254-255.

2. Pervin, F.; Chen, W. W. Mechanically Similar Gel Simulants for Brain Tissues. Dynamic Behavior of Materials 2011, 1, 9-13.

3. Jiang, Y.; Kohara, K.; Hiwada, K. Low Wall Shear Stress in Carotid Arteries in Subjects with Left Ventricular Hypertrophy. Am. J. Hypertens. 2000, 13, 892-898. 